\title{
Preoccupation with a Restricted Pattern of Interest in Modelling Autistic Learning
}

\author{
Lennart Gustafsson ${ }^{1}$ and Andrew P. Papliński ${ }^{2}$ \\ 1 Computer Science and Electrical Engineering, \\ LuleåUniversity of Technology, S-971 87 Luleå, Sweden \\ Lennart.Gustafsson@sm.luth.se \\ 2 Computer Science and Software Engineering, \\ Monash University, Victoria 3800, Australia \\ app@csse.monash.edu . au
}

\begin{abstract}
Autism is a developmental disorder in which attention shifting is known to be restricted. Self-organization of neural networks, conditioned by different attention shifting characteristics is investigated for higher-dimensional stimuli presented to the network from different sources. The attention shifting modes are 1) novelty seeking, 2) attention shift impairment (attention is shifted but with a low probability) and 3) attention is shifted with a preference for a source which has become familiar to the map. The feature maps resulting from self-organization are much the same for modes 1 and 2 but distinctly different for mode 3 , where the maps learn the stimuli from the source with the lowest variability in great detail, at the expense of the other source(s). Detailed learning in narrow fields is a known characteristic of autism.
\end{abstract}

\section{Introduction: Autism, Restricted Attention Shifting}

Autism is a developmental disorder with diagnostic criteria (DSM-IV, 1994 [1]) grouped in three basic categories, impairments in social interaction, impairments in verbal and nonverbal communication, and restricted repetitive and stereotyped patterns of behavior, interests, and activities. Each category is further divided into subcategories, and of particular interest to this paper is the subcategory of the third category which deals with "encompassing preoccupation with one or more stereotyped and restricted patterns of interest". Kanner [2] and Asperger [3] were first to describe autism. Kanner considered an obsessive demand for sameness to be a cardinal feature of autism. This, which obviously is related to the above diagnostic criterion, is also of importance for the topic of this paper.

It is generally agreed that attention shifting is not normal in autism but the underlying cause is a matter of debate with two main hypotheses, a general attention shifting impairment [4], and attention shifting restricted by familiarity preference or novelty avoidance $[5,6,7,8]$. A brief review of these hypotheses can be found in [9]. 
This is a continuation of our earlier work on modelling autism presented in $[9,10,11,12,13]$ where we examined the use of Kohonen Self-Organizing Maps (SOMs) subjected to two-dimensional data. We found that attention shifting restricted by familiarity preference causes the feature map to learn the data from the source with the least variability in its data whereas normal learning, i.e. learning with attention shifts to that source which presents new data, resulted in maps which adapt to the data from both sources. Learning under general attention shifting impairment resulted in maps that were very similar to the maps obtained from normal learning. No dysfunction of the neural networks themselves is present.

In this paper an artificial neural network model of learning is used to show how detailed learning in narrow fields develops when attention shifting between different sources of stimuli is restricted by familiarity preference.

\section{Artificial Neural Networks Used In Modelling}

Our model of autistic learning is based on Kohonen Self-Organizing Maps (SOMs) [14] extended with of the attention shift mechanism. Details of our model were presented in $[11,12,10,13]$. Here we briefly present basic aspects of the model.

It is well known that a Self-Organizing Map is a competitive neural network in which $m$ neurons, each with $p$ synapses are organized in an $l$-dimensional lattice (grid) representing the feature space. Such a neural network performs mapping of a $p$-dimensional input space into the $l$-dimensional feature space. In the examples presented in this paper the dimensionality of the input space is relatively high $(p=19)$ and the feature space is two-dimensional.

In order to make calculations of the distance between weight vectors and stimuli simpler and more biologically plausible we project our $(p-1)-$ dimensional stimuli $\hat{\mathbf{x}}(n)$ onto a unity $p$-dimensional sphere. With all augmented $p$-dimensional stimuli $\mathbf{x}$ and weight vectors located on the hyper-sphere, the distance between them can be measured simply by the post-synaptic activities, that is, by the inner products of weight and stimuli vectors, which is equal to the cosine of the angles between the stimulus and the weight vectors. For the normalised stimuli, in order to keep the weight vectors on the surface of the unity sphere we used the "dot-product" learning law [14].

\section{Source Familiarity Filter and Attention Shift Mechanism}

The block-diagram of the model of autistic learning is presented in Figure 1. The central part is the SOM neural network as described above. At each learning step a stimulus is randomly generated from one of the sources, $S_{1}, \ldots S_{c}$. The attention shifting mechanism determines if that stimulus is presented to the map for learning. 


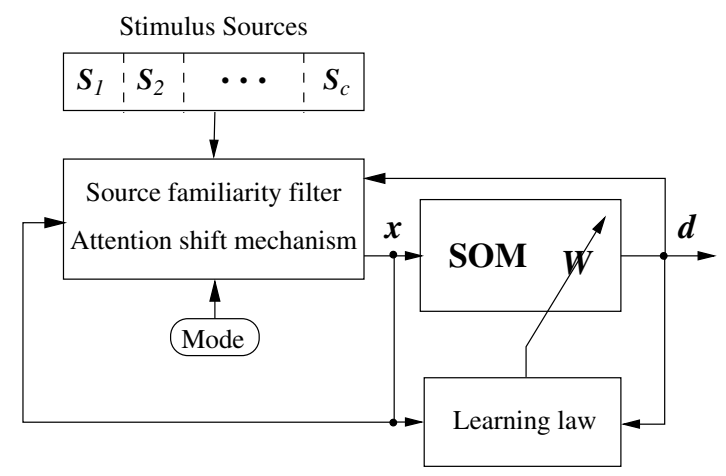

Fig. 1. A block-diagram of the model of autistic learning

In the normal, i.e. the novelty seeking learning mode, attention is shifted to another source if the new stimulus originates from that source (mode 1). In mode 2, general attention shifting impairment learning mode, attention is also shifted to another source if the new stimulus originates from that source, but only with the low probability of $1 \%$ (the results are insensitive to this number).

In mode 3 , with the attention shifting restricted by familiarity preference, attention is shifted to another source if that source presents the next new stimulus, but conditionally, depending on the map's familiarity with that source. The map familiarity to a particular source is measured by the time averaged value of the distance between map nodes and the stimuli. When both sources are unfamiliar to the map, i.e. in the beginning stage of self-organization, attention is shifted to an alternate source if that source presented the next stimulus as in the novelty seeking mode. As the map develops some familiarity with the sources, i.e. the node weights begin to resemble the data, attention is shifted with a higher probability to the source which is most familiar to the map. If the map becomes familiar to two or more sources (the average difference between node weights and the data from the sources becomes smaller than a predetermined small value) then attention is unconditionally shifted.

\section{Modelling Autistic Learning with High-Dimensionality Stimuli}

In the simulations the width of the afferent connections, that is, the dimensionality of the stimuli and weight vectors, is relatively high $(p=19)$. The neural network which is used to model the stimuli consists of sixteen neurons organized in a $4 \times 4$ two-dimensional grid. With such a high dimensionality the feature map cannot be visualised in the input space. However the dimensionality of the feature space, i.e. of the neuronal grid, is still low $(l=2)$ and the feature maps can be illustrated in this space by attaching to each neuron stimuli located in the proximity of the relevant weight vector. 
Table 1. Angular distances in the pairs of the closest animals

\begin{tabular}{|cr|}
\hline \multicolumn{2}{|c|}{ Source A } \\
\hline \hline Horse $\leftrightarrow$ Zebra & 28 \\
Wolf $\leftrightarrow$ Dingo & 55 \\
WSwan $\leftrightarrow$ Bswan & 38 \\
Salmon $\leftrightarrow$ Trout & 36 \\
PBear $\leftrightarrow$ KBear & 50 \\
Rhino $\leftrightarrow$ Hippo & 42 \\
Kangaroo $\leftrightarrow$ Wallaby & 44 \\
Anaconda $\rightarrow$ PBear & 103 \\
Whale $\rightarrow$ Hippo & 406 \\
\hline
\end{tabular}

\begin{tabular}{|cr|}
\hline \multicolumn{2}{|c|}{ Source B } \\
\hline \hline CatEcld $\rightarrow$ catSiam & 9 \\
catstrp $\rightarrow$ catSiam & 9 \\
panther $\leftrightarrow$ leopard & 12 \\
ocelot $\rightarrow$ lynxEur & 41 \\
jaguar $\leftrightarrow$ tiger & 40 \\
lion $\rightarrow$ tiger & 45 \\
\hline \hline \multicolumn{2}{|c|}{ Test cats } \\
\hline \hline catBlck $\rightarrow$ catSiam & 9 \\
catSiam $\rightarrow$ catEcld & 9 \\
snowLprd $\rightarrow$ leopard & 13 \\
lynxEur $\rightarrow$ ocelot & 41 \\
\hline
\end{tabular}

\subsection{Stimuli Specification}

The higher-dimensional stimuli are here chosen to be animals and a number of their characteristics, mostly visual. One source contains animals of widely different kinds: mammals, birds, a reptile and fish. The other source contains only cats. There are animals which stand out and there are other animals that are very similar to each other, in some cases separated only by coloration. The animals are listed below:

Source A: Przewalski's horse, Grevy's zebra, Canis lupus (wolf), Dingo, White (mute) swan, Black swan, Atlantic salmon, Rainbow trout, Polar bear, Kodiak bear, White rhinocerous, Hippopotamus, Grey Western kangaroo, Swamp wallaby, Anaconda, Grey whale

Source B: even colored domestic cat, striped domestic cat, black panther, leopard, ocelot, jaguar, lion tiger.

For investigating the generalization properties acquired by the map during learning we also have four test animals: black domestic cat, Siamese cat, snow leopard and Eurasian lynx. The animals have been characterized by weight, food, locomotion (fins, wings, two legs, four legs), feet (hooves, claws or other), coloration (black, white, even colored, spotted, striped), facial feature (elongated or short nose), aquatic preference and social structure. Each animal is described by eighteen numbers. We project the animal characterization on a 19-dimensional unity sphere as described earlier.

In order to assess the categorization characteristics of the maps it is necessary to establish the "likeness" among these animals. In Table 1 pairs of greatest likeness are presented together with a measure of their angular distance on the hyper-sphere. The first animal is chosen and then the animal that most resembles it is calculated. It is clear from Table 1 that the anaconda and the whale stand out and that the domestic cats are very similar to each other. There are also 
many pairs like horse $\leftrightarrow$ zebra and a group of three similar big cats, jaguar, lion and tiger. A good map of only sixteen nodes would thus assign one node each for the whale and the anaconda, let the domestic cats share one node, let the three big cats share one node and let a number of pairs of animals share one node per pair. In a map formed by attending to Source B only, a good map would assign one node to each of the cats.

\subsection{Learning Results}

The feature map resulting from the normal, novelty seeking learning is shown in Figure $2 \mathbf{a}$. The map consists of the $4 \times 4$ neuronal grid. Each animal is shown at the node with the best match of weights. The angular distance between the animal vector and the node weight vector is shown after the animal name. In the map the number of animals for which a given node is the closest varies from three to zero ("dead neurons"). As expected the whale is represented by its own node with distance 0 , i.e. a perfect match between the animal and the best matching node has been achieved. As also anticipated the three big cats share one node almost in the middle between them. Other animals are likewise represented as anticipated. Four nodes are dead (unassigned). The feature maps resulting from learning with a general attention shifting impairment (mode 2) show the same properties as the maps resulting from the normal, novelty seeking learning; this is shown in Table 2 where statistics from 100 simulations in each learning mode is presented.

The feature map resulting from learning in the attention shifting restricted by familiarity preference mode is shown in Figure $2 \mathbf{b}$. This map, which devel-

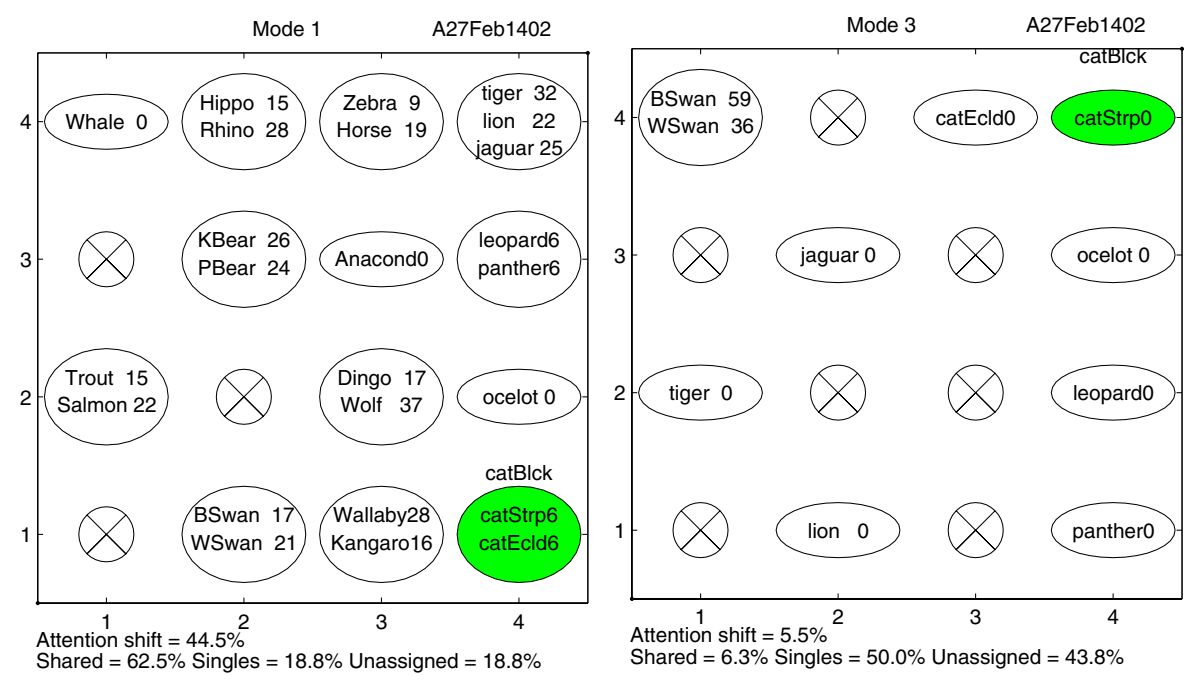

Fig. 2. The feature maps developed in the a. novelty seeking, and b. attention shifting restricted by familiarity preference learning modes. The shaded ovals represent the network response to a test animal 
oped largely after the attention shifting had ceased, reveals the characteristics of detailed learning in a narrow field, that is, the stimuli source with the lowest variability, i.e. the cats, is learned. Every cat is represented by its best matching node and all these best matches are perfect. This is possible because the number of neurons considerably exceeds the number of cats. Several of the nodes marked as "unassigned" have weight vectors which are close to the cats which have been allocated to neighbouring (closest match) neurons. There is also a poor, "chance" learning of one pair from source A.

\subsection{Testing for Generalization}

A good map should have acquired some generalization capability, i.e. when presented with a stimulus it has not learned, it should be represented by a node with weights that are similar if such a node exists. We presented the maps from Figures $2 \mathbf{a}$ and $2 \mathbf{b}$ with the "Black Cat" stimulus which has not been used in learning. In both cases the node closest to this test stimulus is the one allocated to the striped cat, possibly shared with other similar cats. These nodes are shaded in Figure 2.

\subsection{Statistics of Learning}

In Table 2 statistics from one hundred simulations of learning in the three different modes are presented. It is obvious from these statistics that there is no significant difference between the resulting maps from learning in mode 1 and mode 2 but that the resulting maps from learning in mode 3 are altogether different.

Table 2. Statistics from one hundred simulations. The left table presents means and standard deviations of the sums of the animal-closest weight vector distances. The right table presents percentages of the number of attention shifts during learning

\begin{tabular}{|r||r|r|r|}
\hline \multicolumn{4}{|c|}{ Sums of distances } \\
\hline & mode 1 & mode 2 & mode 3 \\
\hline \hline means, non-cats: & 242.50 & 241.18 & 1776.26 \\
means, cats: & 119.47 & 118.98 & 13.22 \\
std, non-cats: & 38.40 & 36.94 & 294.99 \\
std, cats: & 36.23 & 47.94 & 10.50 \\
\hline
\end{tabular}

\begin{tabular}{|r||r|r|r|}
\hline \multicolumn{4}{|c|}{ Attention shifts } \\
\hline & mode 1 & mode 2 & mode 3 \\
\hline \hline mean: & $43.97 \%$ & $0.43 \%$ & $6.05 \%$ \\
std: & $0.44 \%$ & $0.06 \%$ & $0.89 \%$ \\
\hline
\end{tabular}

\section{Conclusion}

Self-organization of feature maps presented with stimuli from more than one source will result in very different maps depending on the rules for attention 
shifting between the sources. In learning under novelty seeking the resulting "normal" map will represent all sources and group stimuli in an "economic" way - similar stimuli will share the same node. Learning with a general attention shifting impairment results in "normal" maps. This does not support the hypothesis that a general attention shifting impairment will cause an autistic development. In learning under familiarity preference - a characteristic wellknown in autism - the resulting map will represent only the source with the lowest variability and will use its representational capacity so that stimuli with very minor differences, such as the coloration of domestic cats, each have their own nodes. The learning which is modelled by such self-organization corresponds to detailed learning in narrow fields, a characteristic well-known in autism.

\section{Acknowledgements}

This work is a result of a cooperation between Luleå University of Technology, Luleå, Sweden and Monash University, Clayton, Australia. We wish to express our appreciation to the Swedish STINT grant scheme and the Monash School of Computer Science and Software Engineering for supporting this cooperation. We also thank the anonymous Reviewers for their inspirational comments.

\section{References}

[1] Diagnostic and statistical manual of mental disorders. 4th ed. American Psychiatric Association (1994) Available from: http://www.psychologynet.org. 1122

[2] Kanner, L.: Autistic disturbances of affective contact. Nervous Child (1943) $217-2501122$

[3] Asperger, H.: Die 'autistischen Psychopathen' im Kindesalter. Arch. Psychiatrie Nervenkrankheiten (1944) 76-136 Translated in Frith U. (ed) (1991): Autism and Asperger Syndrome. Cambridge University Press. 1122

[4] Courchesne, E., Townsend, J., Akshoomoff, N., Saitoh, O., Yeung-Courchesne, R., Lincoln, A., James, H., Haas, R., Schreibman, L., Lau, L.: Impairment in shifting attention in autistic and cerebellar patients. Behavioral Neuroscience 108 (1994) $848-865 \quad 1122$

[5] Kootz, J., Marinelli, B., Cohen, D.: Modulation of response to environmental stimulation in autistic children. Journal of Autism and Developmental Disorders 12 (1982) 185-193 1122

[6] Dawson, G., Meltzoff, A., Osterling, J., Rinaldi, J., Brown, E.: Children with autism fail to orient to naturally occurring social stimuli. Journal of Autism and Developmental Disorders 28 (1998) 479-485 1122

[7] Pascualvaca, D., Fantie, B., Papageorgiou, M., Mirsky, A.: Attentional capacities in children with autism: Is there a general deficit in shifting focus? Journal of Autism and Developmental Disorders 28 (1998) 467-478 1122

[8] Minshew, N., Luna, B., Sweeney, J.: Oculomotor evidence for neocortical systems but not cerebellar dysfunction in autism. Neurology (1999) 917-922 1122

[9] Gustafsson, L., Papliński, A.P.: Self-organization of an artificial neural network subjected to attention shift impairments and novelty avoidance: Implications for the development of autism. Journal of Autism and Developmental Disorder (2002). Submitted for publication. 1122, 1123 
[10] Papliński, A.P., Gustafsson, L.: An attempt in modelling autism using selforganizing maps. In: Proc. 9th Intern. Conf. Neural Information Processing, Singapore (2002) 301-304 1123

[11] Gustafsson, L., Papliński, A.P.: An experiment in modelling learning in autism using self-organizing artificial neural networks. Technical Report 2001/93, School of Comp. Sci. and Soft. Eng., Monash University, Australia (2002) 1123

[12] Gustafsson, L., Papliński, A.P.: Autistic-like detailed learning in a narrow range of stimuli: results from simulations with artificial neural networks restricted by familiarity preference. In: Inaugural World Autism Congress 2002, Melbourne (2002) 1123

[13] Papliński, A.P., Gustafsson, L.: An attempt in modelling autism using selforganizing maps. In: Proc. 9th Intern. Conf. Neural Information Processing, Istanbul (2002) submitted for presentation. 1123

[14] Kohonen, T.: Self-Organising Maps. 3rd edn. Springer-Verlag, Berlin (2001) 1123 